Tine Tjørnhøj-Thomsen: None declared, Torkell Ellingsen Grant/research support from: Novartis 2017 (unrestricted research grant)

DOI: 10.1136/annrheumdis-2021-eular.2076

\section{AB0925-PARE A NARRATIVE REVIEW ASSESSING THE ROLE OF DIETARY SALT AS AN ENVIRONMENTAL RISK FACTOR FOR THE ONSET AND SEVERITY OF RHEUMATOID ARTHRITIS}

S. Ahmed ${ }^{1}$, E. Nikiphorou ${ }^{2,3}$, J. Bayliss ${ }^{4} .{ }^{1}$ Colchester Hospital, Rheumatology, Colchester, United Kingdom; ${ }^{2}$ Kings College London, Centre for Rheumatic Diseases, London, United Kingdom; ${ }^{3}$ Kings College London, Rheumatology Department, London, United Kingdom; ${ }^{4}$ East Suffolk and North Essex NHS Foundation Trust, Library \& Learning Resources, Ipswich, United Kingdom

Background: The role of dietary salt consumption in the etiopathogenesis of Rheumatoid Arthritis (RA), and autoimmune disease in general, has received renewed interest. This has been fueled by the increased prevalence of autoimmune disease worldwide correlating with western diets and heightened consumption of salt rich foods and also studies at the cellular level demonstrating induction of IL 17 producing T helper cells (Th17) by dietary salt.

Objectives: To conduct a narrative review of observational studies and clinical trials on the role of dietary salt as an environmental risk factor for the onset and development of RA.

Methods: A comprehensive search was done of the literature from 2010 to 2021 using the search terms dietary salt and RA; the native interfaces EBSCO and Ovid were used. Databases searched included Pubmed, Embase, EMCare, Medline and CINAHL using a Population, Exposure and Outcome framework; the MESH terms RA, risk factors, nutrition and salt were used. Data was extracted by an independent reviewer.

Results: Out of the 72 studies initially identified, 50 were included in this review. Studies in murine models have demonstrated that high concentrations of sodium chloride promote the differentiation of T helper lymphocytes, via the serum- and glucocorticoid- inducible kinase 1 (SGK1) mediator towards the proinflammatory Th17 driven immune response. Six studies were carried out in human subjects. Study design ranged from cross sectional observational to nested case control studies. Sodium intake amongst participants characterized as having high intake, or being placed in the higher quartiles, ranged from 4.5-5grams per day. 5 out of 6 studies demonstrated that increased dietary salt consumption is associated with earlier onset RA. One study suggested an association between high salt intake and erosive disease at diagnosis and the development of anti-citrullinated protein antibodies (ACPA), although evidence was weak and from a single study only. Another study found that increased consumption of salt was only associated with risk of RA in smokers, highlighting the need to explore confounding variables further.

Conclusion: This narrative review of the literature provides some evidence that supports a role of excess dietary salt consumption as a risk factor for the onset and severity of RA.

Disclosure of Interests: None declared

DOI: 10.1136/annrheumdis-2021-eular.2972

\section{Psychosocial support}

\section{AB0926-PARE IMPACT OF COVID 19 PANDEMIC ON TUNISIAN SPA PATIENTS: PSYCHOLOGICAL STATE AND TREATMENT ADHERENCE}

M. Rachdi ${ }^{1}$, A. Feki ${ }^{2}$, Z. Gassara ${ }^{3}$, S. Ben Jemaa ${ }^{4}$, M. Ghali ${ }^{4}$, M. Ezzeddine ${ }^{4}$, M. H. Kallel ${ }^{4}$, H. Fourati ${ }^{4}$, R. Akrout ${ }^{4}$, S. Baklouti ${ }^{4} .{ }^{1}$ Hedi Chaker Hospital Sfax, Rheumatology, Sfax, Tunisia; ${ }^{2}$ Hedi Chaker Hospital, Rheumatology, Sfax, Tunisia: ${ }^{3}$ Hedi Chaker Hospital, Rheumatology, Sfax, Tunisia: ${ }^{4}$ Hedi Chaker Hospital, Rheumatology, Sfax, Tunisia

Background: The challenge posed by the COVID-19 pandemic may represent an overwhelmingly stressful event for ankylosing spondylitis $(\mathrm{SpA})$ patients and impact their treatment adherence. In response to the COVID-19 pandemic, Tunisia,have adopted community containment to manage the spread of the virus. However, COVID-19 restrictions can alter psychological wellbeing and limit access to treatment for SpA patients.

Objectives: This study aimed to evaluate the impact of COVID-19 pandemic on psychological health and treatment adherence on Tunisian SpA patients.

Methods: This is a cross sectional study including patients with SpA (ASAS criteria). A survey comprising questions about adherence to stay home warnings; the obligation to go outside for work; satisfaction with the medical support or information received for COVID-19; showing up to medical check-ups, proper use of the medications; medications that the patient stopped taking.
Anxious and depressive symptoms were assessed using the Arabic version of Hospital Anxiety and Depression Scale (HADS) questionnaire.

Results: We included thirty patients. the average age was: 39,7 years-old and the sex ratio was: $13,3.75 \%$ of patients were married. The SpA was axial in $25 \%$, peripheral in $20 \%$, and both in $55 \%$. Most patients had a moderate activity and the mean activity scores were: BASDAI $=2.60$, ASDAScrp:2.65

$38 \%$ of patients were on biologics, $36 \%$ on sulfasalazine and NSAIDs and 26 $\%$ on NSAIDs only.

It seemed that significant number of patients strictly adhered to stay home warnings (>89\%) only $11 \%$ were obliged to go out for work during general lockdown while only $24 \%$ adhered to it after general lockdown.

Most of the patient $78 \%$ were not satisfied with the medical support or information about COVID 19. $88 \%$ of patients requested information from TV while 10 $\%$ requested it from social media and $2 \%$ from relatives and friends working in health care field.

After the outbreak, $23 \%$ of the patients who had a scheduled chek-up visit attended the appointment as it was before. The remaining either 'did not want to come' $(43 \%)$, wanted to come but could not contact anyone in the hospital $(11 \%)$, was advised to postpone their visits $(10 \%)$, or couldn't find means of transport (13\%).A significant number of patients decreased or skipped their dose $(69 \%)$, while only $13 \%$ continued their medications and $16 \%$ stopped taking NSAIDs.

Biological DMARDs(anti-TNF agents) were the most frequent drugs which patients decreased their dose, skipped or stopped taking $33 \%$. sulfasalazine and NSAIDs were least likely $17 \%$ to be skipped or stopped.

$43 \%$ of patients Had a HADS anxiety level more than or equal to $11: 87 \%$ women and $13 \%$ men. The highest anxiety scores were found among patients aged less than 45 years old $(87 \%)$ married with children $.32 \%$ of patients had a HADS depression level more than or equal to $11: 54 \%$ women and $44 \%$ men. the highest depression scores were found among patients aged less than 45 years old married with children

No significant relationship was found between anxiety and depression levels regarding biologic treatment.

Conclusion: Our results suggest that patients with $\mathrm{SpA}$ were less likely to comply strictly to 'stay home' restrictions, most probably due to the male predominance and relatively younger age. Additionally, we noticed that SpA patients treated with anti-TNF agents were the patients that regular drug use had been considerably disrupted.

COVID 19 pandemic has heightened the need to care for patients with $\mathrm{SpA}$ in an increasingly virtual environment.

Additionally, we found that being female, having a lower level of education, having a child, living in a crowded family is correlated to higher levels of anxiety and depression.

\section{REFERENCES:}

[1] Smarr KL, Keefer AL. Measures of depression and depressive symptoms: Beck Depression Inventory-II (BDI-II),Hospital Anxiety and Depression Scale (HADS), and Patient Health Questionnaire-9 (PHQ-9) Arthritis care.

Acknowledgements: I would like to express my special thanks of gratitude to Rheumatology department of Hedi Chaker Hospital Sfax.

Disclosure of Interests: None declared

DOI: 10.1136/annrheumdis-2021-eular.3950

\section{Work and rehabilitation}

\section{AB0927-PARE WORK PRODUCTIVITY AND ACTIVITY IMPAIRMENT IN PATIENTS WITH SAPHO SYNDROME}

L. Gong ${ }^{1}$, C. Li ${ }^{2} .{ }^{1}$ Peking Union Medical College Hospital, Institute of Clinical Medicine, Beijing, China: ${ }^{2}$ Peking Union Medical College Hospital, Department of Traditional Chinese Medicine, Beijing, China

Background: Synovitis, acne, pustulosis, hyperostosis, and osteitis (SAPHO) syndrome is a rare disease, characterized by osteoarticular and cutaneous manifestations. Osteitis and hyperostosis are regarded as the core pathophysiological changes of SAPHO syndrome [1], which may lead to bone pain and loss of motor function. The Work Productivity and Activity Impairment (WPAI) questionnaire is an instrument to measure the impact of the disease on work productivity and activity, subsequently adapted for ankylosing spondylitis [2] rheumatoid arthritis [3], irritable bowel syndrome [4], and other chronic diseases [5]. However, no study has investigated the work productivity of patients with SAPHO syndrome.

Objectives: The purpose of this study is to give an overview of work productivity loss in SAPHO patients through the work productivity and activity impairmen (WPAI) questionnaire and investigate the relationship between the WPAI and other disease-related indicators. 
Methods: Patients for this cross-sectional study were recruited from Peking Union Medical College Hospital (Beijing, China). The questionnaires incorporating the WPAI were administered, along with demographic data, disease-specific measures, and general health variables. The construct validity of WPAI was evaluated by the correlations between WPAI outcomes and other measures. Wilcoxon rank-sum tests and non-parametric Kruskal-Wallis tests were used for comparison of WPAI outcomes between known-groups.

Results: A total of 376 patients were included and 201 patients (53.5\%) were employed. The median (interquartile range [IQR]) of absenteeism, presenteeism, work productivity loss and activity impairment were 0\% (0-13\%), 20\% (0-40\%), $20 \%(0-52 \%)$ and $30 \%(0-50 \%)$, respectively. All WPAI outcomes showed moderate to strong correlations to other generic and disease-specific measures (|r| $=0.43-0.75$ ), except for absenteeism. Increasing disease activity and worse health status are significantly associated with higher impairment of work productivity and activity.

Conclusion: This study highlights the negative effects of SAPHO syndrome on patients' work productivity and activity, indicating a good construct validity and discriminative ability of WPAI. For reducing the economic burden, it is important to improve the patients' work productivity and daily activity with active intervention.
REFERENCES:

[1] Liu S, Tang M, Cao Y, Li C. Synovitis, acne, pustulosis, hyperostosis, and osteitis syndrome: review and update. Therapeutic advances in musculoskeletal disease. 2020;12:1759720x20912865.

[2] Reilly MC, Gooch KL, Wong RL, Kupper H, van der Heijde D. Validity, reliability and responsiveness of the Work Productivity and Activity Impairment Questionnaire in ankylosing spondylitis. Rheumatology (Oxford, England). 2010;49(4):812-819.

[3] Zhang W, Bansback N, Boonen A, Young A, Singh A, Anis AH. Validity of the work productivity and activity impairment questionnaire--general health version in patients with rheumatoid arthritis. Arthritis research \& therapy. 2010;12(5):R177.

[4] Frandemark A, Tornblom H, Jakobsson S, Simren M. Work Productivity and Activity Impairment in Irritable Bowel Syndrome (IBS): A Multifaceted Problem. The American journal of gastroenterology. 2018;113(10):1540-1549.

[5] Enns MW, Bernstein CN, Kroeker K, Graff L, Walker JR, Lix LM, et al. The association of fatigue, pain, depression and anxiety with work and activity impairment in immune mediated inflammatory diseases. PloS one. 2018;13(6):e0198975.

Disclosure of Interests: None declared

DOI: 10.1136/annrheumdis-2021-eular.484 